\title{
Enhanced Food-Related Motivation after Bilateral Lesions of the Subthalamic Nucleus
}

\author{
Christelle Baunez, ${ }^{1}$ Marianne Amalric, ${ }^{1}$ and Trevor W. Robbins ${ }^{2}$ \\ 1 Laboratoire de Neurobiologie Cellulaire et Fonctionnelle, Centre National de la Recherche Scientifique, 13402 Marseille \\ cedex 20, France, and 2Department of Experimental Psychology, University of Cambridge, CB2 3EB, Cambridge, \\ United Kingdom
}

\begin{abstract}
Although inactivation of the subthalamic nucleus (STN) has beneficial effects on motor symptoms of parkinsonism, little is known of possible actions on nonmotor symptoms of cognition or mood. Here, we used several forms of converging evidence to show that STN lesions can enhance behavioral motivation. Thus, bilateral fiber-sparing lesions of the STN in rats reduced the time required to eat a standard number of food reward pellets, without affecting food intake, and altered performance on a number of behavioral measures consistent with enhanced motivation for food. Thus, STN-lesioned rats showed greater
\end{abstract}

levels of locomotor activity conditioned to food presentation, enhanced control over responding by food-related conditioned reinforcers, and a higher breaking point associated with elevated rate of lever press under a progressive ratio schedule of reinforcement. These results reveal a new functional role schedule for STN, possibly because of its involvement in ventral, as well as dorsal, striatal circuitry and are relevant to the therapeutic effects of STN stimulation in Parkinson's disease.

Key words: basal ganglia; conditioned behavior; consummatory behavior; incentive motivation; progressive ratio; feeding
The subthalamic nucleus (STN) is part of the basal ganglia, a group of structures classically considered to be involved in motor functions, because various lesions affecting these cerebral structures induce obvious motor deficits, such as Parkinson's disease (PD). Experimental data have suggested that hyperactivity of the STN could be responsible for some of the deficits in parkinsonism. Recently, the STN has proven to be a promising target for the surgical treatment of PD. Lesions or high-frequency stimulation of STN in PD patients (Limousin et al., 1995) and in animal models of PD (Bergman et al., 1990, Benazzouz et al., 1993; Baunez et al., 1995; Henderson et al., 1999) indeed improve motor symptoms. However, inactivation of the STN in these conditions can also impair additional aspects of behavior (Baunez et al., 1995; Henderson et al., 1999; Trépanier et al., 2000; Krack et al., 2001). Because the STN is a key structure involved in the classical parallel loops linking the basal ganglia to various cortical areas (Alexander et al., 1986) with motor, associative, and limbic components, the STN might influence a wide range of behavioral functions. Thus, it is important to characterize the behavioral role of the STN further.

Initial studies of STN lesions focused on obvious motor effects. For example, a consequence of selective STN lesions in humans and nonhuman primates induced ballism (Whittier, 1947; Whittier and Mettler, 1949). In rats, bilateral STN lesions increase

\footnotetext{
Received July 9, 2001; revised Oct. 10, 2001; accepted Oct. 19, 2001.

This study was supported by the Centre National de la Recherche Scientifique (CNRS), 5th Programme-Cadre de Recherche et de Développement Technologique. Funding Grant QLK6-1999-02173, the Fondation France Parkinson (M.A.), CNRS-Royal Society funding, and the Wellcome Trust (T.W.R.). C.B. was supported by Human Frontier Science Program Long-Term Fellowship LT-358/1996 at the beginning of the work. We thank Dr. M. Cador for helpful discussion, Y. Darbaky for helping with the consumption test, D. Terramorsi for taking care of the animals, and Eric Dubois and Céline Martin for computer programming assistance.

Correspondence should be addressed to Christelle Baunez, Laboratoire de Neurobiologie Cellulaire et Fonctionnelle, Centre National de la Recherche Scientifique, 31 Chemin Joseph Aiguier, 13402 Marseille cedex 20, France. E-mail: baunez@Incf.cnrs-mrs.fr.

Copyright (ㄷ) 2002 Society for Neuroscience $\quad 0270-6474 / 02 / 220562-07 \$ 15.00 / 0$
}

anticipatory responding in a reaction time task (Baunez et al., 1995), whereas unilateral lesions induce either rotation (Kafetzopoulos and Papadopoulos, 1983) or postural impairment (Phillips et al., 1998). Only recently have the cognitive roles of the STN been investigated. STN excitotoxic lesions affect visual attentional performance (Baunez and Robbins, 1997, 1999), as well as mechanisms of response selection and working memory (Baunez et al., 2001). However, there has been no detailed investigation of possible roles of the STN in motivational processes, although perseverative behavior induced by STN lesions was ameliorated by prefeeding (Baunez and Robbins, 1997).

The present study is the first to investigate directly the involvement of STN in various aspects of motivation. We measured effects of STN lesions on both consummatory behavior (latency to eat 100 food pellets) and appetitive behavior, assessed in three ways: (1) locomotor activity conditioned to food presentation, a direct measure of incentive motivation (Campbell and Sheffield 1953; Jones et al., 1990); (2) the efficacy of a food-related conditioned reinforcer to support new learning (Robbins et al., 1989); and (3) responding under a progressive ratio (PR) schedule of reinforcement in which rats are required to make increasingly large numbers of responses to obtain standard food reinforcement (Hodos, 1961).

\section{MATERIALS AND METHODS}

\section{Animals}

Male Long-Evans Rats (Janvier, Le Genest St. Isles, France) and Lister hooded (Charles River, Kent, UK) [for the conditioned reinforcement (CR) experiment] were housed in pairs and maintained on a $12 \mathrm{hr}$ light/dark cycle (lights on at 7:00 A.M.). During the experiments using food reinforcement, they were kept at $80-85 \%$ of their free feeding weight by restricting their food to $15-17 \mathrm{gm} / \mathrm{d}$ per rat. Water was provided ad libitum, except during experimental sessions. All procedures were conducted in accordance with either the requirements of the United Kingdom Animals (Scientific Procedures) Act 1986 or the French Agriculture and Forestry Ministry decree 87-849. 


\section{Apparatus}

Eight $26.5 \times 22 \times 20 \mathrm{~cm}$ standard operant boxes (Med Associates Inc., St. Albans, VT) were used. Four boxes had a retractable lever and a stimulus light located above the food magazine. Next to the lever, a magazine $(60 \times 50 \mathrm{~mm})$ connected with a food pellet dispenser provided access to reward. The apparatus and on-line data collection was controlled by a computer and an interface (MedPC; Med Associates Inc.). Four other boxes had two retractable levers and a stimulus light located in the magazine located between the levers and connected with a sucrose solution dipper providing access to reward. The apparatus and on-line data collection was controlled by a computer (Acorn Computers, Cambridge, UK) and an interface (Paul Fray Ltd., Cambridge, UK). All boxes were equipped with photocell beams at $15 \mathrm{~mm}$ from the entrance of the magazine. Breaks of these beams registered frequency and duration of magazine entries.

Sixteen individual $370 \times 227 \times 235 \mathrm{~mm}$ Perspex locomotor activity cages with a grid floor were used. Each cage was traversed by two parallel infrared photocell beams located at the front $(100 \mathrm{~mm}$ from the entrance) and at the rear ( $100 \mathrm{~mm}$ from the end) of the cage. Beam breaks were recorded in 10 min bins on-line by a computer equipped with an Imetronic (Bordeaux, France) extension.

\section{Behavioral procedure}

\section{Food consumption test}

Animals (sham, $n=13$; STN, $n=18$; some of which had undergone measures of conditioned locomotor activity and others responding under the progressive ratio) were placed, one after the other, in a plastic home cage measuring $42 \times 25 \times 12 \mathrm{~cm}$. In one corner, $10045 \mathrm{mg}$ sweet Noyes-type pellets were placed, and the time taken for the rat to eat the pellets was timed in seconds. The box was then cleaned with alcohol and left for a few minutes before the next rat was placed in the box with another 100 pellets. Animals from sham and STN groups were tested in a random order.

Another group of animals (sham, $n=7 ; \mathrm{STN}, n=9$ ) was used to test consumption by measuring the quantity of food eaten in a fixed time duration. One week after surgery, while they were fed ad libitum, all rats were placed individually in the locomotor activity cages with a determined amount of chow. Absorbent paper was placed on the floor to prevent food falling through the grid floor. The amount of food eaten in grams $( \pm$ SEM) was measured after $30 \mathrm{~min}, 60 \mathrm{~min}$, and at the end of the session (120 min), correcting for any spillage. A few days later, the same experiment was performed, replacing lab chow by sucrose food pellets in the individual cages. In individual home cages, the total intake of lab chow was also measured after $24 \mathrm{hr}$ for 3 consecutive days and then averaged. The same animals were then food-deprived for 1 week, during which they were fed only with $15 \mathrm{gm} / \mathrm{d}$. Under this food restriction regimen, their intake was assessed again in the locomotor activity cages for $2 \mathrm{hr}$. On one day, a fixed amount of lab chow was placed, and the amount consumed was measured after 30,60 , and $120 \mathrm{~min}$. On a different day, the same procedure was followed with sucrose pellets.

\section{Conditioned locomotor activity}

All testing was conducted from 10:00 A.M. to 12:00 A.M., during the light period and followed a schedule described previously to test effects of basal forebrain cholinergic lesions (Olmstead et al., 1998).

\section{Habituation}

Eighteen rats (sham, $n=11$; STN lesions, $n=7$ ) were placed in the activity cages for $120 \mathrm{~min}$ at the same time each day until stable baseline activity was established. During this phase, feeding (restricted to 15 $\mathrm{gm} / \mathrm{rat}$ ) was done in home cages at varying and unpredictable intervals after testing $(2-6 \mathrm{hr})$.

\section{Conditioning}

During this phase, rats were fed in the activity cages. The food (in the same restricted amount) was presented $30 \mathrm{~min}$ after the recording of locomotor activity had started. The animals remained in the cages for 90 min after delivery of the food (total of $120 \mathrm{~min}$ session). The beam breaks were recorded during the $30 \mathrm{~min}$ prefeeding period, over 10 sessions. No feeding occurred in the home cages.

\section{Extinction}

During this phase, feeding was switched back to the home cages, in similar conditions to those of habituation. This phase lasted until activity returned to baseline levels.

\section{Prefeeding}

During this phase, the locomotor activity was assessed when rats were prefed. Before the test, rats were given $1 \mathrm{hr}$ access to food ad libitum in their home cage. After this $1 \mathrm{hr}$ period, rats were placed in locomotor activity cages for $120 \mathrm{~min}$.

\section{Conditioned reinforcement}

Training. Eleven rats underwent a Pavlovian conditioning procedure in which presentation of a conditioned stimulus (CS) (5 sec illumination of the tray light and house light off) preceded the unconditioned stimulus (US) ( 5 sec elevation of the sucrose solution dipper). The CS-US pairing was presented 30 times per session at random variable intervals varying such that the session would last $\sim 30 \mathrm{~min}$. Twenty sessions were required before stable performance was attained. This was evaluated as a ratio calculated as the mean number of magazine entries during the CS period as a percentage of the mean number of magazine entries during a comparable interval of the variable interval period ( $5 \mathrm{sec} \times 30$ trials). All rats underwent then surgery (sham, $n=7$; STN, $n=6$ ) and were given 1 week recovery period. They were then given three to four retraining sessions to reestablish the baseline before the start of the testing.

Testing. Sucrose was no longer available. Two levers were introduced into the test box, and depression of one lever [no conditioned reinforcer (NCR)] had no programmed consequences, whereas depression of the second lever (CR) resulted in the presentation of the CS (under a random ratio 2 schedule). Assignment of CR and NCR levers was counterbalanced within groups to avoid side-preference effects. Because rats were tested in extinction, they were not tested for more than four sessions. The number of responses on each lever and number of magazine entries were recorded during the 30 min sessions.

\section{Progressive ratio}

As described previously (Eagle et al., 1999), rats (sham, $n=8$; STN, $n=$ 7) were initially trained to press a lever to obtain a food pellet under a continuous reinforcement schedule. Every lever press had as a consequence the onset of a light located above the magazine and the delivery of a food pellet. Additional lever presses had no consequence until the rat's nose was detected in the magazine. The session ended when 100 pellets were delivered or $30 \mathrm{~min}$ had elapsed. After 10 sessions under this schedule, all rats had acquired stable performance and were moved to the PR schedule. Rats were then reinforced for lever pressing under an arithmetically increasing fixed-ratio (FR) schedule in steps of 5, with three repetitions of each step (i.e., $1,1,1,5,5,5,10,10,10,15,15,15 \ldots$ ). The lever press that completed each FR within this PR schedule induced the onset of the light above the magazine and the delivery of a single pellet. The light remained on until the rat's nose was detected in the magazine. Additional lever presses (perseverative lever presses) had no consequences but were counted. A session ended if the rat failed to press the lever for 5 consecutive min or 90 min had elapsed. Training on the PR schedule was undertaken after surgery only and for 10 consecutive sessions (one session per day). For each session, the value of the last completed ratio was recorded, as well as the perseverative lever presses, the number of magazine entries, and the duration of the session. Therefore, the rate of lever presses could be estimated by summation of total lever presses (appropriate plus perseverative lever presses) divided by the session duration expressed in minutes.

\section{Statistical analysis}

The data were analyzed using ANOVA with the Statview program (Statview 5; SAS Institute, Cary, NC). The results are expressed as means for each of the variables (i.e., time required to eat 100 pellets, number of lever presses on the CR lever, NCR lever, last ratio reached in the PR procedure, session duration, etc.) in the different groups of animals.

For each variable, the data were submitted to mixed-design ANOVAs, with group (sham vs STN lesions) as the between-subject factors and sessions as the within-subject factors when appropriate. When significant effects were found, post hoc comparisons between means were made using simple main effects analysis.

\section{Surgery}

All animals were anesthetized with xylazine $(15 \mathrm{mg} / \mathrm{kg}$, i.m.) and ketamine $(100 \mathrm{mg} / \mathrm{kg}$, i.m.) and secured in a Kopf stereotaxic apparatus (Phymep, Paris, France). Twenty rats received bilateral injection of ibotenic acid $(9.4 \mu \mathrm{g} / \mu \mathrm{l}$, i.e., $53 \mathrm{~mm}$; Research Biochemicals, Illkirch, 

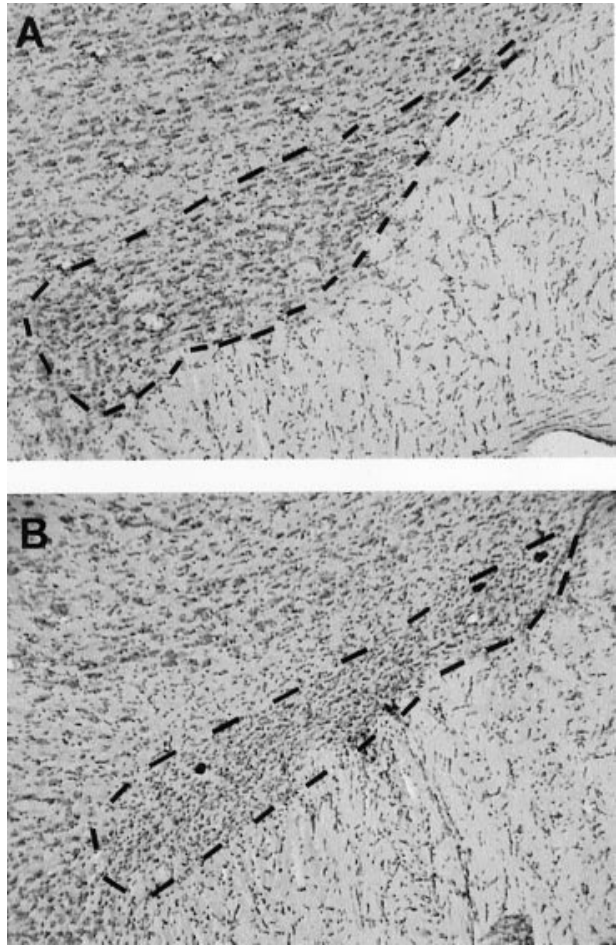

Figure 1. Photomicrographs of frontal sections stained with cresyl violet, at the level of the STN outlined by dashed lines, illustrating an STN in a sham $(A)$ and in a lesioned $(B)$ animal.

France), and 26 rats received the vehicle alone ( $0.1 \mathrm{M}$ phosphate buffer). The volume injected was $0.5 \mu \mathrm{l}$ per side inf used over 3 min using a $10 \mu \mathrm{l}$ Hamilton microsyringe, connected by Tygon tubing fitted to the 30 gauge stainless steel injector needles, and fixed on a micropump calibrated to deliver the exact volume over a period of $3 \mathrm{~min}$.

The injection coordinates were taken as the average of interaural and bregma coordinates from the atlas of Paxinos and Watson (1986) (from the bregma): anteroposterior, $-3.8 \mathrm{~mm}$; lateral, $+2.4 \mathrm{~mm}$; dorsoventral, $-8.35 \mathrm{~mm}$ (from skull); incisor bar set at $-3 \mathrm{~mm}$. As mentioned previously (Baunez et al., 1995), while recovering from anesthesia, STN rats exhibit a short-lasting self-biting behavior that completely disappears when they wake up. Therefore, protection of the paws was provided by bandaging and removed immediately after the animals had recovered from anesthesia.

\section{Histology}

After completion of the behavioral testing, all of the animals were perfused under deep chloral hydrate anesthesia $(400 \mathrm{mg} / \mathrm{kg}$, i.p.) with $4 \%$ paraformaldehyde solution through the left cardiac ventricle. The brains were removed and post-fixed overnight at $4{ }^{\circ} \mathrm{C}$ in the same fixative. They were then put into a $10 \%$ sucrose solution to be further frozen. Frontal 30- to $40-\mu \mathrm{m}$-thick sections of the STN were cut by use of a cryostat (Leica, Nussloch, Germany) and collected for cresyl violet staining. Only rats showing a bilateral lesion of the STN, characterized by loss of body cells and intense gliosis reaction leading to a shrinkage of the STN, as illustrated in Figure 1, were included in the data analysis. Lesions were restricted to the STN, and, in most cases, few neurons were spared in the very lateral tip. The extent of the lesion is shown in Figure 1. Two animals with mislocated lesions were discarded after the histological examination conducted blind to the behavioral findings. Their performance in the CR experiment was in fact different from that of rats with accurate STN lesions.

\section{RESULTS}

\section{Consummatory behavior}

Rats with bilateral lesions of the STN were faster to eat 100 food pellets than controls (mean time to eat in seconds, $788 \pm 57$ vs $1343 \pm 178$; group, $\left.F_{(1,29)}=12.21 ; p<0.01\right)$. The mean $\pm \mathrm{SEM}$ body weights of the two groups, when measured under food restriction were as follows: sham, $401 \pm 15.28 \mathrm{gm}$; STN, $384 \pm$ $10.41 \mathrm{gm}$. When measured after $10 \mathrm{~d}$ of ad libitum feeding, the mean body weight average was $427 \pm 16.77$ and $421 \pm 11.5$ gm for the sham and STN groups, respectively. Although there was a significant effect of the feeding across both groups (ANOVA; $\left.F_{(1,14)}=77.43 ; p<0.01\right)$, there was no significant group $\times$ feeding interaction $\left(F_{(1,14)}=2.46 ; p>0.05\right)$.

Table 1 shows the mean \pm SEM food intake for the STN and sham groups for short $(2 \mathrm{hr})$ and long $(24 \mathrm{hr})$ consumption tests. For the $2 \mathrm{hr}$ consumption test, food intake is also shown when standard lab chow or sucrose pellets are proposed under either sated or food-deprived conditions. Separate ANOVAs for the 2 and $24 \mathrm{hr}$ revealed no significant main effect of STN lesion in either case ( $2 \mathrm{hr}$ sated condition, $F_{(1,14)}=0.09$; food-deprived condition, $\left.F_{(1,14)}=3.99 ; 24 \mathrm{hr}, F_{(1,14)}=0.12\right)$, although there was a nonsignificant trend for STN rats to exhibit less consumption (Table 1) in food-deprived conditions. There were also no significant interactions of lesion with either food type or food deprivation conditions (three-way ANOVA; all $F_{(1,14)}$ values $<1.8$; $p>0.05)$. For the $24 \mathrm{hr}$ consumption, repeated measures over 3 consecutive days showed no difference between sated STNlesioned rats and sated sham rats in their food intake (group effect, $\left.F_{(1,14)}=0.12 ; p>0.05\right)$. This was not affected over the $3 \mathrm{~d}$ (day effect, $\left.F_{(2,28)}=0.27 ; p>0.05\right)$.

\section{Conditioned responses to food reward expectation}

\section{Reward-related learning: conditioned locomotor activity}

This task allows the measurement of anticipatory locomotor responses elicited by expectation of food reward in various phases: initial conditioning (i.e., Pavlovian learning), extinction (omission of food reward), and after prefeeding.

In the very first phases of exposure to a novel environment, STN-lesioned rats were more active than control rats when food deprived $\left(F_{(1,16)}=12.06 ; p<0.01\right)$, although this effect disappeared with repeated exposures to this environment (no significant group effect for the last four sessions) (Fig. 2A). During the next stage, when food was provided in the locomotor activity cages after $30 \mathrm{~min}$, no difference between groups could be found during the preceding $30 \mathrm{~min}$ of the first session $(p>0.05)$ because animals could not predict the presentation of food pellets in the cages. However, from the second day of this conditioning phase, animals increased their activity over the sessions, as an expression of expectation of the food presentation (sessions, $\left.F_{(9,144)}=38.38 ; p<0.01\right)$, this effect being stronger in the STN group (group $\times$ sessions interaction, $F_{(9,144)}=3.33 ; p<0.01$ ) (Fig. $2 B$ ). This response diminished when food was no longer presented in the locomotor activity cages but given in the animal holding room instead. Although extinction was slower in the STN group (group $\times$ sessions interaction, $F_{(13,208)}=3.84 ; p<0.01$ ), no significant difference was recorded between the two groups for the last three sessions (sessions $11-14, p>0.05$ ) (Fig. 2C, left). When all animals were prefed (after access to food ad libitum for $1 \mathrm{hr}$ before testing), no significant difference was recorded between the two groups (group, $F_{(1,16)}=0.42 ; p>0.05$ ) (Fig. $2 C$, right).

\section{Conditioned reinforcement}

Conditioned reinforcement reflects the process whereby a previously conditioned stimulus acts as the reinforcer for an instrumental action (Mackintosh, 1974). This test allows, in two separate phases, the measurement of the following: (1) the strength of 
Table 1. Food intake for the STN and sham groups

\begin{tabular}{|c|c|c|c|c|c|}
\hline & \multicolumn{4}{|c|}{$2 \mathrm{hr}$ consumption $(\mathrm{gm} \pm \mathrm{SEM})$} & \multirow{3}{*}{$\begin{array}{l}24 \mathrm{hr} \text { consumption }(\mathrm{gm} \pm \mathrm{SEM}) \\
\text { Standard lab chow } \\
\text { Sated }\end{array}$} \\
\hline & \multicolumn{2}{|c|}{ Standard lab chow } & \multicolumn{2}{|c|}{ Sucrose pellets } & \\
\hline & Sated & Food deprived & Sated & Food deprived & \\
\hline Sham $(n=7)$ & $4.36 \pm 1.18$ & $9.86 \pm 1.05$ & $2.87 \pm 1.34$ & $14.6 \pm 1.31$ & $21.68 \pm 1.41$ \\
\hline $\operatorname{STN}(n=9)$ & $4.17 \pm 0.87$ & $7.7 \pm 0.64$ & $2.39 \pm 0.67$ & $12.19 \pm 1.23$ & $21.13 \pm 1.07$ \\
\hline
\end{tabular}

Pavlovian conditioning (i.e., association between a CS (a light) and a US (sucrose solution); and (2) the rewarding properties of the stimulus that was paired previously with food reward.

After recovery from surgery, the rats were given four sessions of the Pavlovian conditioning phase to check for the effects of the lesion on the CS-US pairing. There was no significant difference between the sham- and STN-lesioned rats during this phase. As shown in Figure 3, when rats have the choice between the CR lever (having for consequence the occurrence of the CS associated previously with the sucrose solution) and the NCR lever (having no consequence), they press preferentially on the CR lever (lever effect, $F_{(1,18)}=25.90 ; p<0.01$ ). The number of lever presses on the CR lever was significantly higher in the STN lesion group than in the sham control group (group, $F_{(1,18)}=6.71 ; p<$ $0.05)$ (group $\times$ lever interaction; $\left.F_{(1,18)}=5.16 ; p<0.05\right)$.

\section{Evaluation of the reinforcing property of food (progressive ratio schedule)}

Under progressive ratios, the measure of the last ratio reached (also called "breaking point") allows to assess the amount of effort a rat is willing to expend to obtain the food reinforcer. Under this procedure, rats with bilateral STN lesions reached a significantly higher breaking point than sham control animals (group effect, $\left.F_{(1,13)}=6.47 ; p<0.05\right)($ Fig. $4 A)$. STN-lesioned rats exhibited a higher rate of lever presses (group effect, $F_{(1,13)}=$ 4.73; $p<0.05$ ) (Fig. 4B), worked longer, and completed the session after the sham rats (group effect, $\left.F_{(1,13)}=8.74 ; p<0.05\right)$ (data not shown).

\section{DISCUSSION}

In this series of studies, we obtained converging results from several different paradigms for measuring food-motivated responding to show that bilateral lesions of the STN can enhance these aspects of appetitive motivation, thus providing a novel perspective on the functional interactions of this nucleus within the outflow circuitry of the basal ganglia. Rats with lesions of the STN consumed food reward pellets more quickly, exhibited heightened activity conditioned to food presentation, showed an exaggeration of the conditioned reinforcing properties of stimuli formerly predictive of food delivery, and increased breaking points on a progressive ratio schedule designed to assess motivation to work for food reward. The results may have some clinical significance in view of reports of effects of STN high-frequency stimulation altering mood, and also feeding, in PD patients.

The behavioral analysis required the use of several paradigms to demonstrate the generality of the enhanced appetitive motivation in STN-lesioned rats. The conditioning of locomotor activity to the periodic presentation of food has traditionally been regarded as a powerful demonstration of incentive motivation for food, i.e., a state in which general increases in behavior are generated by a central motive state of eating (Bolles, 1972; Bindra, 1978). Thus, the greatly elevated levels of locomotor
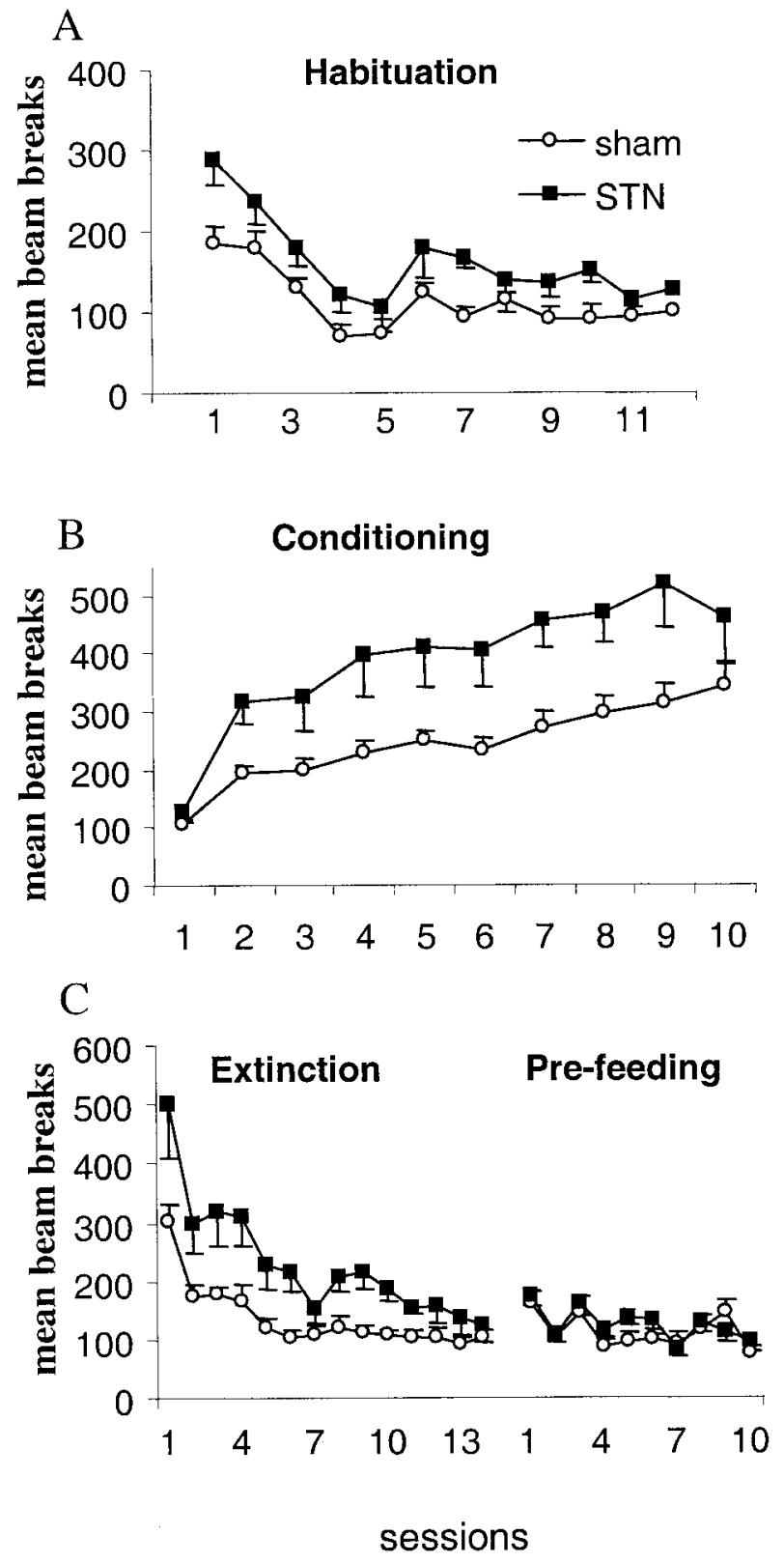

Figure 2. Effects of bilateral STN lesions on conditioned locomotor response for food over the various phases: habituation $(A)$, conditioning $(B)$, and extinction and prefeeding $(C)$. Each point represents the mean \pm SEM number of cell-beam crosses during the first $30 \mathrm{~min}$ for the various daily sessions (abcissas). The activity is illustrated for sham control animals (open circles; $n=11$ ) and for STN-lesioned rats (filled squares; $n=7)$. 


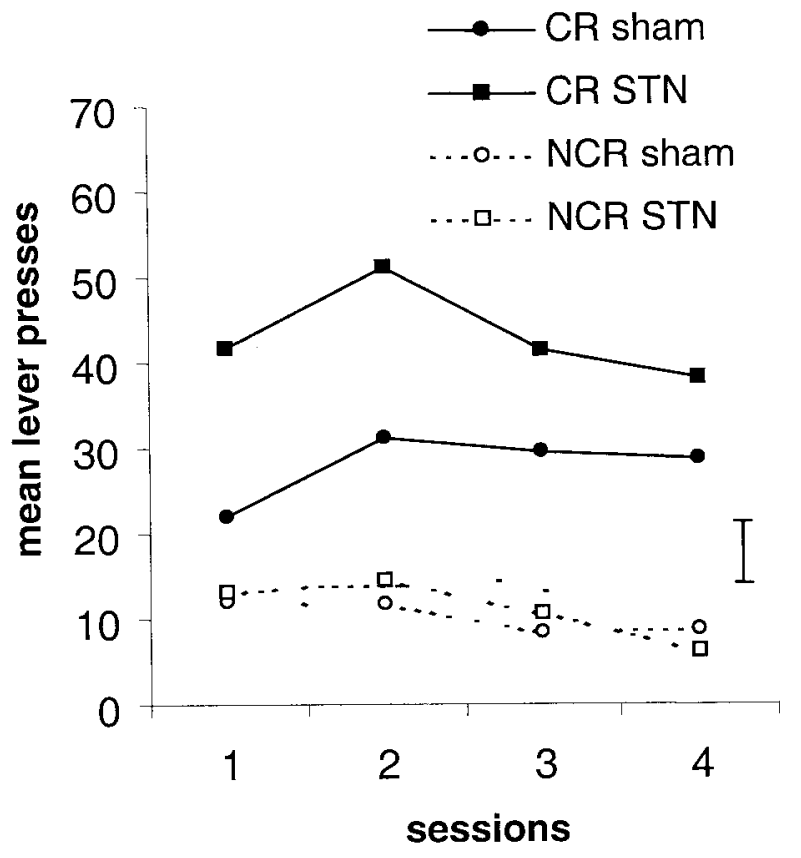

Figure 3. Effects of post-training bilateral STN lesions on acquisition of responding with a conditioned reinforcer (CR). The number of lever presses on the lever producing the CS (CR lever) and on the control lever (NCR) are illustrated for sham ( filled and open circles, respectively; $n=$ 7 ) and lesioned ( filled and open squares, respectively; $n=4$ ) animals. Vertical bar, SEM.

activity in anticipation of food presentation in STN-lesioned rats is consistent with heightened incentive motivation. The demonstration of enhanced Pavlovian conditioning was complemented by the finding that food-related conditioned reinforcers exert greater control over instrumental responding in the STN-lesioned animals. Finally, in a situation in which such influences may combine to influence the amount of effort invested by the animals in obtaining food, STN-lesioned rats showed greater propensity to work for food reward under a progressive ratio schedule. This was shown from the fact that sham-operated controls ceased operant responding before rats with STN lesions as the required number of responses for each standard delivery of food was progressively increased.

These effects are unlikely simply to reflect nonspecific motor disinhibition, as has been suggested previously to account for increases in impulsive responding in reaction time settings (Baunez et al., 1995; Phillips and Brown, 1999). This is because the increases in response output observed here after STN lesions are situation dependent. For example, STN lesion-induced locomotor hyperactivity is restricted to novel test situations, not extending over the full 120 min testing period or repeated sessions of testing. Moreover, the hyperactivity was contingent on the presence of state of feeding motivation: prefeeding or extinction removed the effect.

When STN-lesioned rats were tested after pretraining to approach a food magazine during a food-predictive CS, they did not exhibit nonspecific tendencies to approach when the CS was not presented, again demonstrating a lack of nonspecific behavioral disinhibition. Instrumental responding for conditioned reinforcement involved a choice situation, in which pressing a control lever had no effect but was equivalent in motoric terms to responding on the lever providing conditioned reinforcement. The STN le-
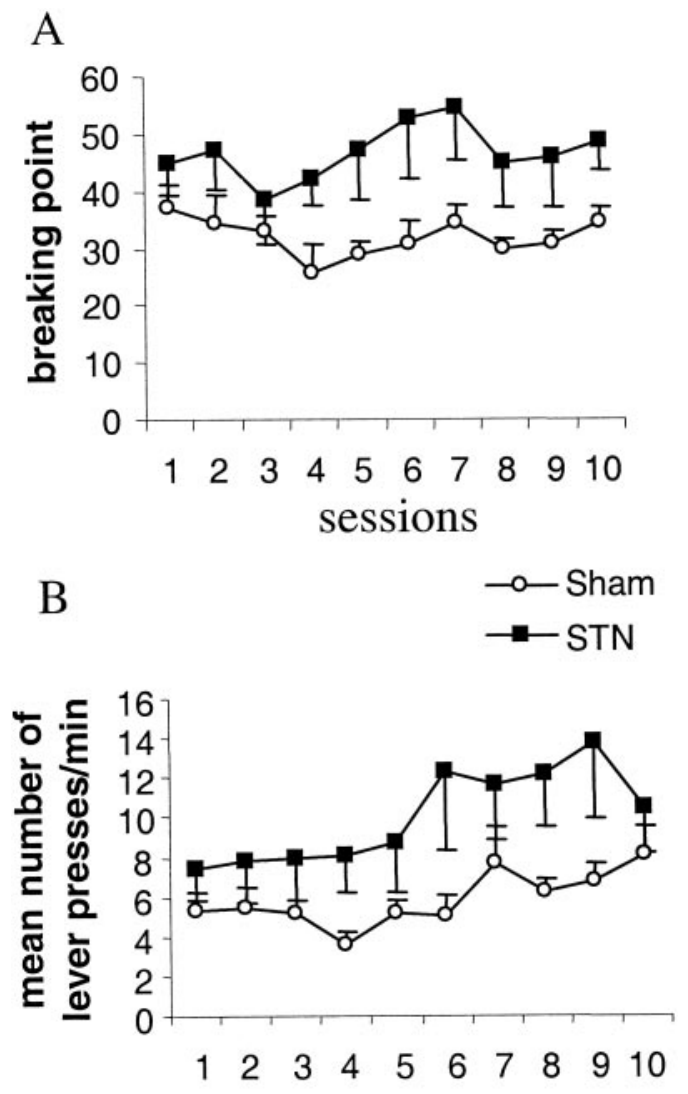

sessions

Figure 4. Effects of bilateral STN lesions on performance in the PR task evaluated by the mean \pm SEM last ratio reached during the session (i.e., breaking point) $(A)$ and the rate of responding measured as the total number of lever presses per minute $( \pm$ SEM $)(B)$. Sham control animals $(n=8$; open circles) and STN-lesioned animals $(n=7$; filled squares $)$ performance is illustrated over 10 sessions.

sions selectively enhanced responding on this conditioned reinforcement lever but did not affect responding on the control lever. It seems most parsimonious to conclude that the STN-lesioned rats were exhibiting higher levels of motivation with respect to food-related behaviors than simple motor disinhibition. This conclusion is also supported by the finding that these rats ate food pellets more quickly in a consumption test. It is unlikely that the rats have primary changes in hunger, because their weight was equivalent to that of controls when food deprived and they gained weight normally when allowed ad libitum access to food. The present experiments measuring food intake directly argue against an effect of STN lesion on primary motivation or hunger. Overall, however, it is apparent that STN lesions affect food-seeking, appetitive rather than consummatory behavior.

As in previous studies (Baunez et al., 1995; Baunez and Robbins, 1997, 1999; Baunez et al., 2001), the bilateral STN lesion selectively damaged all sectors of the STN bilaterally, sparing only a few cells in the most lateral portions of the nucleus. Lesions were restricted to the STN, but some rats exhibited sparing of the nucleus and were excluded from the behavioral analyses. In all cases, these animals performed similarly to sham-operated controls. 
Therefore, it appears that the behavioral effects arose from damage to the STN, which would disrupt outflow from the striatum for behaviors generally associated with ventral, rather than dorsal, striatal function. Previous work has indicated the important role of the STN as part of the "indirect pathway" implicated in modulating the balanced output from the dorsal striatum at the level of the globus pallidus, internal segment (GPi) substantia nigra (Wichmann et al., 1994; Baunez and Amalric, 1996). Those studies in general have emphasized the role of the STN in the control of response output, for example, in reaction time settings, consistent with the hypothesis that the dorsal striatum is concerned with the control of motor output and some aspects of cognitive function (Graybiel et al., 1994; Baunez et al., 1995). The present study, in contrast, has studied some aspects of behavior that have been linked with the functioning of the nucleus accumbens, for example, responding for conditioned reinforcement (Beninger et al., 1981; Robbins et al., 1989, Kelley and Delfs, 1991), conditioned locomotor activity (Jones et al., 1990), and schedule-controlled responding with progressive ratios (Bowman and Brown, 1998; Eagle et al., 1999). For example, responding with conditioned reinforcement is relatively less affected by dopamine depletion in the dorsal, compared with ventral, striatum (Taylor and Robbins, 1986). Moreover, although PR responding is affected by dorsal striatal damage, this generally reflects motor or executive, rather than motivational, effects (Eagle et al., 1999). Thus, the excitotoxic lesions of the lateral striatum produced perseverative responding and some slowing of responding but did not affect breaking point per se. Moreover, medial striatal lesions mainly slowed the latency to collect earned food rather than affecting the breaking point for instrumental responding. In contrast, Bowman and Brown (1998) showed that excitotoxic lesions of the nucleus accumbens increased the breaking point, analogous to the effects of STN lesions. Finally, consistent with this analysis, the control of locomotor activity is generally associated with the ventral striatal circuitry rather than that from dorsal striatum (Kelly et al., 1975). However, the increased feeding seen after STN lesions might be associated with dorsal (Pisa, 1988), as well as ventral, striatal (Kelley, 1999) outflow because regions within the rat striatum are implicated in the control of consummatory behavior. More recently, the excitatory STN projections to the ventral pallidum have been shown to play a major role in ventral pallidal activity (Turner et al., 2001). Thus, some of the effects observed after STN lesions on feeding behavior may be mediated through the ventral pallidum, in which pharmacological manipulations have been shown to affect feeding behavior (Stratford et al., 1999). Overall, our data are consistent with the STN being implicated in the expression of several components of motivation, including both consummatory and appetitive aspects, under control of both the dorsal and ventral striatum.

These new results reflect the anatomical relations that have been highlighted recently to exist between the ventral striatum and STN. Thus, the STN also participates in a "ventral loop" encompassing the nucleus accumbens, ventral pallidum, and STN, which is analogous to the indirect pathway of the dorsal striatum. More specifically, the core region of the nucleus accumbens projects to that ventral pallidal area, which projects to the medial part of the STN (Maurice et al., 1999). There are successive GABAergic connections from the core region to the STN (via the ventral pallidum), suggesting that lesions of the STN should simulate in part effects of core lesions. However, although our data on locomotor activity and the progressive ratio performance after STN lesions are consistent with this simple picture (Bow- man and Brown, 1998; Parkinson et al., 1999), it does not fit for the present effects of STN lesions on instrumental responding for conditioned reinforcement (Parkinson et al., 1999) or food pellet consumption, the latter being more sensitive to manipulations of the nucleus accumbens shell (Kelley, 1999). Evidently, the functional role of the STN is not simply as an output relay of the nucleus accumbens. This complexity is also evident in comparing effects of STN lesions with those of dorsal striatal lesions on reaction time or attentional tasks. It is possible that the additional cortical projections to the STN exert additional influences, which may explain these functional differences.

The present experimental findings may be relevant in part to certain clinical observations of effects of STN lesions or stimulation on mood, feeding, and other forms of motivation (Trillet et al., 1995). For example, a recent case report showed that an STN infarct induced hypersexuality (Absher et al., 2000). Although there are no reports of changes of feeding motivation after STN lesions, pallidotomies or deep brain stimulation affecting GPi or STN are well known to enhance appetite and induce weight gain in PD patients (Lang et al., 1997; Moro et al., 1999; Ondo et al., 2000). Finally, STN stimulation has also been reported to have beneficial or detrimental effects on mood (Ardouin et al., 2000; Trépanier et al., 2000; Krack et al., 2001), apparently not secondary to simple motor effects. Our results suggest that it may be profitable to reexamine these motivational effects of STN stimulation quite closely, not only for possible benefits in PD, but also in the treatments of abulic states (Brown and Pluck, 2000) resulting from basal ganglia damage.

\section{REFERENCES}

Absher JR, Vogt BA, Clark DG, Flowers DL, Gorman DG, Keyes JW, Wood FB (2000) Hypersexuality and hemiballism due to subthalamic infarction. Neuropsychiatry Neuropsychol Behav Neurol 13:220-229.

Alexander GE, DeLong MR, Strick PL (1986) Parallel organization of functionally segregated circuits linking basal ganglia and cortex. Annu Rev Neurosci 9:357-381.

Ardouin C, Pillon B, Peiffer E, Bejjani P, Limousin P, Damier P, Arnulf I, Benabid AL, Agid Y, Pollak P (2000) Neuropsychological changes between "off" and "on" STN or GPi stimulation in Parkinson's disease. Neurology 55:411-418.

Baunez C, Amalric M (1996) Evidence for functional differences between entopeduncular nucleus and substantia nigra: effects of APV (DL-2-amino-5-phosphonovaleric acid) microinfusion on reaction time performance in the rat. Eur J Neurosci 8:1972-1982.

Baunez C, Robbins TW (1997) Bilateral lesions of the subthalamic nucleus induce multiple deficits in an attentional task in rats. Eur J Neurosci 9:2086-2099.

Baunez C, Robbins TW (1999) Effects of dopamine depletion of the dorsal striatum and further interaction with subthalamic nucleus lesions in an attentional task in the rat. Neuroscience 92:1343-1356.

Baunez C, Nieoullon A, Amalric M (1995) In a rat model of parkinsonism, lesions of the subthalamic nucleus reverse increases of reaction time, but induce a dramatic premature responding deficit. J Neurosci 15:6531-6541.

Baunez C, Humby T, Eagle DM, Ryan LJ, Dunnett SB, Robbins TW (2001) Effects of STN lesions on simple vs choice reaction time tasks in the rat: preserved motor readiness, but impaired response selection. Eur J Neurosci 13:1609-1616.

Benazzouz A, Gross C, Féger J, Boraud T, Bioulac B (1993) Reversal of rigidity and improvement in motor performance by subthalamic highfrequency stimulation in MPTP-treated monkey. Eur J Neurosci 5:382-389.

Beninger RJ, Hanson DR, Phillips AG (1981) The acquisition of responding with conditioned reinforcement: effects of cocaine, $(+)$ amphetamine and pipradrol. Br J Pharmacol 74:149-154.

Bergman H, Wichmann T, DeLong MR (1990) Reversal of experimental parkinsonism by lesion of the subthalamic nucleus. Science 249:1436-1438.

Bindra D (1978) How adaptive behavior is produced: a perceptualmotivational alternative to response-reinforcement. Behav Brain Sci 1:41-91.

Bolles RC (1972) Reinforcement, expectancy, and learning. Psychol Rev 79:394-409.

Bowman EM, Brown VJ (1998) Effects of excitotoxic lesions of the rat 
ventral striatum on the perception of reward cost. Exp Brain Res 123:439-448.

Brown RG, Pluck G (2000) Negative symptoms: the "pathology" of motivation and goal-directed behaviour. Trends Neurosci 23:412-417.

Campbell BA, Sheffield FD (1953) Relation of random activity to food deprivation. J Comp Physiol Psychol 46:320-322.

Eagle DM, Humby T, Dunnett SB, Robbins TW (1999) Effects of regional striatal lesions on motor, motivational, and executive aspects of progressive-ratio performance in rats. Behav Neurosci 113:718-731.

Graybiel AM, Aosaki T, Flaherty AW, Kimura M (1994) The basal ganglia and adaptive motor control. Science 265:1826-1831.

Henderson JM, Annett LE, Ryan LJ, Chiang W, Hidaka S, Torres EM, Dunnett SB (1999) Subthalamic nucleus lesions induce deficits as well as benefits in the hemiparkinsonian rat. Eur J Neurosci 11:2749-2757.

Hodos W (1961) Progressive ratio as a measure of reward strength. Science 134:943-944.

Jones GH, Marsden CA, Robbins TW (1990) Increased sensitivity to amphetamine and reward-related stimuli following social isolation in rats: possible disruption of dopamine-dependent mechanisms of the nucleus accumbens. Psychopharmacology 102:364-372.

Kafetzopoulos E, Papadopoulos G (1983) Turning behavior after unilateral lesion of the subthalamic nucleus in the rat. Behav Brain Res $8: 217-223$.

Kelley AE (1999) Functional specificity of ventral striatal compartments in appetitive behaviors. Ann NY Acad Sci 877:71-90.

Kelley AE, Delfs JM (1991) Dopamine and conditioned reinforcement. I. Differential effects of amphetamine microinjections into striatal subregions. Psychopharmacology 103:187-196.

Kelly PH, Seviour PW, Iversen SD (1975) Amphetamine and apomorphine responses in the rat following 6-OHDA lesions of the nucleus accumbens septi and corpus striatum. Brain Res 94:507-522.

Krack P, Ardouin C, Funkiewiez A, Caputo E, Benazzouz A, Benabid AL, Pollak P (2001) What is the influence of subthalamic nucleus stimulation on the limbic loop? In: Basal ganglia and thalamus in health and movement disorders (Kultas-Ilinsky K, Ilinsky IA, eds), pp 333340. New York: Kluwer.

Lang AE, Lozano A, Tasker R, Duff J, Saint-Cyr J, Trépanier L (1997) Neuropsychological and behavioral changes and weight gain after medial pallidotomy. Ann Neurol 41:834-836.

Limousin P, Pollak P, Benazzouz A, Hoffmann D, Lebas JF, Broussole E, Perret JE, Benabid AL (1995) Effects on parkinsonian signs and symptoms of bilateral subthalamic nucleus stimulation. Lancet 345:91-95.

Mackintosh NJ (1974) The psychology of animal learning. London: Academic.

Maurice N, Deniau JM, Glowinski J, Thierry AM (1999) Relationships between the prefrontal cortex and the basal ganglia in the rat: physiology of the cortico-nigral circuits. J Neurosci 19:4674-4681.

Moro E, Scerrati M, Romito LM, Roselli R, Tonali P, Albanese A (1999) Chronic subthalamic nucleus stimulation reduces medication requirements in Parkinson's disease. Neurology 53:85-90.
Olmstead MC, Robbins TW, Everitt BJ (1998) Basal forebrain cholinergic lesions enhance conditioned approach responses to stimuli predictive of food. Behav Neurosci 112:611-629.

Ondo WG, Ben-Aire L, Jankovic J, Lai E, Contant C, Grossman R (2000) Weight gain following unilateral pallidotomy in Parkinson's disease. Acta Neurol Scand 101:79-84.

Parkinson JA, Olmstead MC, Burns LH, Robbins TW, Everitt BJ (1999) Dissociation in effects of lesions of the nucleus accumbens core and shell on appetitive Pavlovian approach behavior and the potentiation of conditioned reinforcement and locomotor activity by $d$-amphetamine. J Neurosci 19:2401-2411.

Paxinos G, Watson C (1986) The rat brain in stereotaxic coordinates, Ed 2. Sydney: Academic.

Phillips JM, Brown VJ (1999) Reaction time performance following unilateral striatal dopamine depletion and lesions of the subthalamic nucleus in the rat. Eur J Neurosci 11:1003-1010.

Phillips JM, Latimer MP, Gupta S, Winn P, Brown VJ (1998) Excitotoxic lesions of the subthalamic nucleus ameliorate asymmetry induced by striatal dopamine depletion in the rat. Behav Brain Res 90:73-77.

Pisa M (1988) Motor somatotopy in the striatum of rat: manipulation, biting and gait. Behav Brain Res 27:21-35.

Robbins TW, Cador M, Taylor JR, Everitt BJ (1989) Limbic-striatal interactions in reward-related processes. Neurosci Biobehav Rev $13: 155-162$

Stratford TR, Kelley AE, Simansky KJ (1999) Blockade of GABAA receptors in the medial ventral pallidum elicits feeding in satiated rats. Brain Res 825:199-203.

Taylor JR, Robbins TW (1986) 6-hydroxydopamine lesions of the nucleus accumbens, but not the caudate nucleus, attenuate enhanced responding with reward-related stimuli produced by intra-accumbens $d$-amphetamine. Psychopharmacology 90:390-397.

Trépanier LL, Kumar R, Lozano AM, Lang AE, Saint-Cyr JA (2000) Neuropsychological outcome of G Pi pallidotomy and GPi or STN deep brain stimulation in Parkinson's disease. Brain Cogn 42:324-347.

Trillet M, Vighetto A, Croisile B, Charles N, Aimard G (1995) Hemiballismus with logorrhea and thymo-affective disinhibition caused by hematoma of the left subthalamic nucleus. Rev Neurol 151:416-419.

Turner MS, Lavin A, Grace AA, Napier TC (2001) Regulation of limbic information outflow by the subthalamic nucleus: excitatory amino acid projections to the ventral pallidum. J Neurosci 21:2820-2832.

Whittier JR (1947) Ballism and the subthalamic nucleus (nucleus hypothalamicus; corpus Luysii). Arch Neurol Psychiatry 58:672-692.

Whittier JR, Mettler FA (1949) Studies of the subthalamus of the rhesus monkey. II. Hyperkinesia and other physiologic effects of subthalamic lesions, with special references to the subthalamic nucleus of Luys. J Comp Neurol 90:319-372.

Wichmann T, Bergman H, DeLong MR (1994) The primate subthalamic nucleus. I. Functional properties in intact animals. J Neurophysiol $72: 494-506$. 